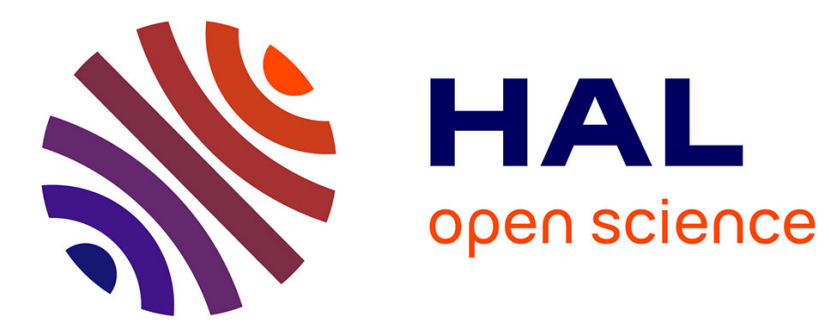

\title{
Inertial coalescence of droplets on a partially wetting substrate
}

Yi Sui, Marco Maglio, Peter D. M. Spelt, Dominique Legendre, Hang Ding

\section{To cite this version:}

Yi Sui, Marco Maglio, Peter D. M. Spelt, Dominique Legendre, Hang Ding. Inertial coalescence of droplets on a partially wetting substrate. Physics of Fluids, 2013, 25, pp.1-7, 101701. $10.1063 / 1.4824108$. hal-00919847

\section{HAL Id: hal-00919847 https://hal.science/hal-00919847}

Submitted on 17 Dec 2013

HAL is a multi-disciplinary open access archive for the deposit and dissemination of scientific research documents, whether they are published or not. The documents may come from teaching and research institutions in France or abroad, or from public or private research centers.
L'archive ouverte pluridisciplinaire HAL, est destinée au dépôt et à la diffusion de documents scientifiques de niveau recherche, publiés ou non, émanant des établissements d'enseignement et de recherche français ou étrangers, des laboratoires publics ou privés. 


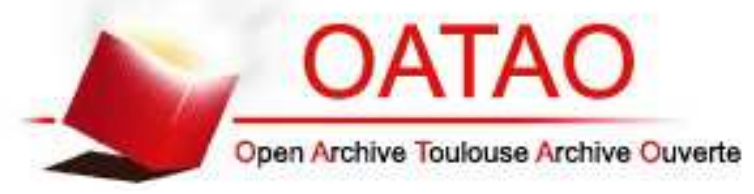

\section{Open Archive TOULOUSE Archive Ouverte (OATAO)}

OATAO is an open access repository that collects the work of Toulouse researchers and makes it freely available over the web where possible.

This is an author-deposited version published in : http://oatao.univ-toulouse.fr/ Eprints ID : 10541

To link to this article :

DOI: $10.1063 / 1.4824108$

URL : $\underline{\text { http://dx.doi.org/10.1063/1.4824108 }}$

\section{To cite this version :}

Sui, Yi and Maglio, Marco and Spelt, D. M. Peter and Legendre, Dominique and Ding, Hang Inertial coalescence of droplets on a partially wetting substrate. (2013) Physics of Fluids, vol. 25. pp. 17. ISSN 1070-6631

Any correspondance concerning this service should be sent to the repository administrator: staff-oatao@listes-diff.inp-toulouse.fr 


\title{
Inertial coalescence of droplets on a partially wetting substrate
}

\author{
Yi Sui, ${ }^{1}$ Marco Maglio, ${ }^{2}$ Peter D. M. Spelt, ${ }^{3}$ Dominique Legendre, ${ }^{2}$ \\ and Hang Ding 4 \\ ${ }^{1}$ School of Engineering and Materials Science, Queen Mary University of London, \\ London E1 4NS, United Kingdom \\ ${ }^{2}$ Institut de Mécanique des Fluides de Toulouse (IMFT), Université de Toulouse, \\ CNRS-INPT-UPS, France \\ ${ }^{3}$ Laboratoire de Mécanique des Fluides \& d'Acoustique (LMFA), CNRS, École Centrale de \\ Lyon, Ecully, France and Département Mécanique, Université Claude Bernard Lyon 1, \\ Villeurbanne, France \\ ${ }^{4}$ Department of Modern Mechanics, University of Science and Technology of China, \\ Hefei 230027, China
}

\begin{abstract}
We consider the growth rate of the height of the connecting bridge in rapid surfacetension-driven coalescence of two identical droplets attached on a partially wetting substrate. For a wide range of contact angle values, the height of the bridge grows with time following a power law with a universal exponent of $2 / 3$, up to a threshold time, beyond which a $1 / 2$ exponent results, that is known for coalescence of freelysuspended droplets. In a narrow range of contact angle values close to $90^{\circ}$, this threshold time rapidly vanishes and a $1 / 2$ exponent results for a $90^{\circ}$ contact angle. The argument is confirmed by three-dimensional numerical simulations based on a diffuse interface method with adaptive mesh refinement and a volume-of-fluid method.
\end{abstract}

Droplet coalescence is an important problem from both a fundamental and an applied points of view. Industrial applications include sintering, ${ }^{1}$ two-phase separation, ${ }^{2}$ and microfluidic actuators. ${ }^{3}$ The coalescence of droplets on a partially wetting substrate (illustrated in Fig. 1) has been studied mostly in a slow, viscous regime, corresponding to a sufficiently large value of an Ohnesorge number, $O h=\mu /(\rho \gamma R)^{1 / 2}$ (where $\mu$ is the liquid viscosity, $\gamma$ the coefficient of surface tension, and $R$ a drop radius). There, the width of the liquid bridge ( $W$ in Fig. 1) grows as $t^{1 / 2}$ for infinitesimal contact angles, which has been demonstrated to result from a simple mass balance model that assumes a constant velocity into the liquid bridge and that the effect of the increase in bridge height on the width is negligible. ${ }^{4}$ Experimental studies have confirmed this result ${ }^{5,6}$ and show that the result carries over to large contact angles ${ }^{6}$ and to low-viscosity liquids. ${ }^{7}$ The corresponding rate at which the height of the liquid bridge for slow coalescence is generally different, Narhe et al. ${ }^{5}$ observed experimentally and predicted from a force balance that in an early stage of slow coalescence the bridge height increases linearly with time, and that the proportionality factor scales quadratically with the contact angle. Lee et al. ${ }^{6}$ identified from their experiments a power-law exponent with the value in the range 0.5-0.9 that increases (as does the proportionality factor in the power law) with the contact angle, and at late times a deviation from a single power law altogether. ${ }^{6}$ HernándezSánchez et al. ${ }^{8}$ identified a unity-value exponent, consistent with Narhe et al. ${ }^{5}$ but they found that the proportionality factor scales with the fourth power of the contact angle. We investigate herein rapid coalescence instead, with the main objective of studying the rate at which the height of the liquid bridge grows.

The growth rate of the height of the liquid bridge in an inertial regime can be predicted by considering the symmetry $(x, z)$ plane depicted in Fig. 1, crucially extending an analysis of Eggers et $a l .{ }^{9}$ for coalescence of freely suspended inviscid droplets. The pressure jump across the interface 

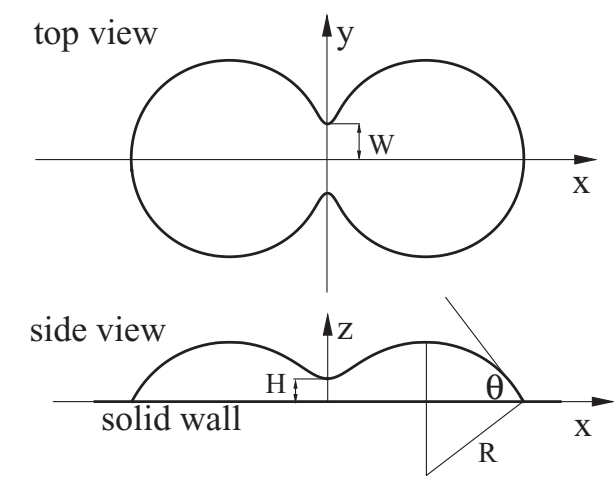

FIG. 1. Coalescence of two droplets on a flat solid substrate.

is estimated on one hand as $\rho(d H / d t)^{2}$, and on the other as $\gamma / L$, where $L$ is the radius of the interface curvature in the middle cross sectional $(x, z)$ plane and can be estimated by the distance between the symmetry $(y, z)$ plane to the surface of the initial droplet, resulting in

$$
L(z)=R \sin \theta-\left(R^{2}-(z+R \cos \theta)^{2}\right)^{1 / 2},
$$

here we have defined $R$ as the radius of curvature of the initial drops, and $\theta$ as the contact angle (shown in Fig. 1). Equating the dynamical pressure $\rho(d H / d t)^{2}$ and the capillary pressure $\gamma / L$, we obtain

$$
\left(1-\left(\frac{1}{\sin ^{2} \theta}-\left(\zeta+\frac{1}{\tan \theta}\right)^{2}\right)^{1 / 2}\right)\left(\frac{d \zeta}{d \tau}\right)^{2}=1,
$$

in terms of $\zeta=H / R \sin \theta$ and $\tau=\left(t-t_{c}\right)\left(\gamma /\left(\rho R^{3} \sin ^{3} \theta\right)\right)^{1 / 2}$, where $t_{c}$ is the time at the instance of coalescence. The main trends in the behaviour of the solution can be identified from a Taylor series expansion (truncated after the first two terms) for small $\zeta$ of the first part in brackets in Eq. (2),

$$
\left(\frac{\zeta}{\tan \theta}+\frac{\zeta^{2}}{2}\left(1+\frac{1}{\tan ^{2} \theta}\right)\right)\left(\frac{d \zeta}{d \tau}\right)^{2}=1
$$

Although this equation can be solved analytically, the result is an implicit nonlinear equation for $\zeta(\tau)$, but the main conclusions are readily drawn from Eq. (3). Evidently, at $\theta=90^{\circ}$, the analysis coincides with that for coalescence of freely suspended droplets (without a wall) and gives $\zeta \sim \tau^{1 / 2}$, which is consistent with that of Eggers et al. ${ }^{9}$

In the generalization to arbitrary contact angles, however, the first-order term in the Taylor series (in Eq. (3)) dominates to give $\zeta \sim \tau^{2 / 3}$ until $\zeta \sim \sin 2 \theta$ when the second-order term becomes important, well beyond which the $\tau^{1 / 2}$ regime is expected. Hence, the $2 / 3$ regime contracts to zero at $\theta=90^{\circ}$ but is otherwise dominant over a large part of the coalescence process. In fact, the maximum value of $\zeta$ (corresponding to the bridge height reaching the top of the drops) is $(1-\cos \theta) / \sin \theta$, which is reached before a transition to the $1 / 2$ regime at $\zeta \sim \sin 2 \theta$ if $\theta<68.53^{\circ}$. So the exponent in a power law is not dependent on the contact angle, but the time range over which an exponent of $1 / 2$ or $2 / 3$ can be observed. This is confirmed by the numerical solution of the full model, Eq. (2), as is seen in Fig. 2: the result for $90^{\circ}$ serves as an upper bound for other angles. It is also seen from these results that at late times, the slope drops somewhat below 1/2; higher-order contributions (of order $n)$ to the above-mentioned Taylor series give rise to a reduction in the power to $(2 /(2+n))$.

For this inertial regime to commence, the instantaneous Reynolds number $\rho H(d H / d t) / \mu$ should be $\gg 1$. The result $\zeta=\tan ^{1 / 3} \theta \tau^{2 / 3}$ obtained from Eq. (3) is therefore consistent with inertia/capillarydominated flow as long as $\tau \gg O h^{3} /\left(\sin ^{3 / 2} \theta \tan ^{2} \theta\right)$ until $\zeta \sim \sin 2 \theta$; the regime $\zeta=(2 \tau / b)^{1 / 2}$ (with $\left.b \equiv\left(1+1 / \tan ^{2} \theta\right)^{1 / 2} / \sqrt{2}\right)$ requires $O h^{2} \ll 2 \sin \theta /\left(1+1 / \tan ^{2} \theta\right)$.

To test this model, we first compare against prior experiments for rapid coalescence, ${ }^{7}$ in Fig. 3. Of course, these prior experiments are necessarily limited from the point of view of the 




FIG. 2. Numerical solutions of Eq. (2) for (from top to bottom) $\theta=90^{\circ}, 89^{\circ}, 87^{\circ}, 80^{\circ}, 45^{\circ}$, and $5^{\circ}$. The top of the drops has been reached $(L=R \sin \theta$ corresponding to $\zeta=(1-\cos \theta) / \sin \theta)$ at the end point of each curve.

present analysis, in that they do not include the range of contact angles that are now of main interest here, which is close to $90^{\circ}$. Nevertheless, it is encouraging to see the experimental data agree well with the present theory, which contains no adjusted parameter, although the uncertainty in the data is such that precise confirmation is not possible.

We have therefore also conducted tests against numerical simulations of droplet coalescence on a substrate that are based on the full 3D Navier-Stokes equations. The approach resolves the flow in the liquid as well as in the surrounding fluid. Although computational methods for two-phase flows are generally well-established, the physical modelling of the vicinity of moving contact lines and its accurate numerical representation remain challenging subjects. ${ }^{10}$ We present here results obtained with two independent methods that allow us to assess the sensitivity of the simulation results to the contact-line model. One of these approaches is based on a diffuse-interface (DI) method, ${ }^{11,12}$ with newly incorporated adaptive mesh refinement function; ${ }^{13,14}$ in the following, we shall indicate by " $n$ levels of refinement" that the finest grid spacing is $1 / 2^{n-1}$ times the coarsest grid spacing. The DI method naturally regulates the stress singularity at the contact line by considering the fluid interface as a diffused layer. The second method used adopts a volume-of-fluid (VOF) approach. ${ }^{15,16}$ In the version of the VOF method employed in the present study, there is no interface reconstruction but the interface is limited to 2 to 3 grid cells, thanks to an accurate flux-corrected transport algorithm. The shear stress singularity at the wall is removed by using the Navier slip boundary condition.

The sensitivity of the results of these simulations to the mesh resolution and numerical method is first investigated in Fig. 4. The dimensionless neck height is shown versus dimensionless time

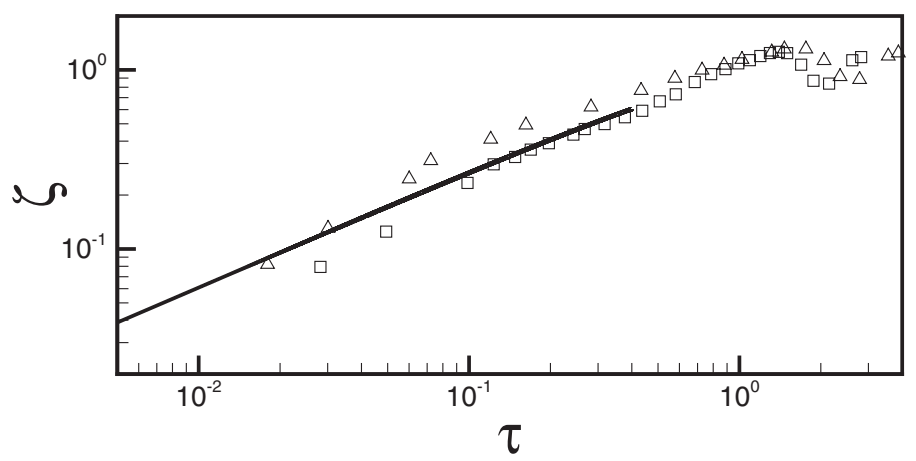

FIG. 3. Comparison of Eq. (2) (solid line) against the experiments of Ref. 7 (symbols) for the bridge height versus time. The theoretical curve is terminated when the bridge height reaches the top of the droplet as explained in the text. In the experiments distilled water droplets with density of $1000 \mathrm{~kg} / \mathrm{m}^{3}$, viscosity of $1.07 \mathrm{mPa} \mathrm{s}$, surface tension coefficient of $0.074 \mathrm{~N} / \mathrm{m}$, and contact angle of $\theta=64^{\circ}$ were used. Droplet footprint radius is $R=1.61 \mathrm{~mm}$ (triangles), $2.3 \mathrm{~mm}$ (squares), corresponding to $O h=0.0029$ and 0.0025 , respectively. 


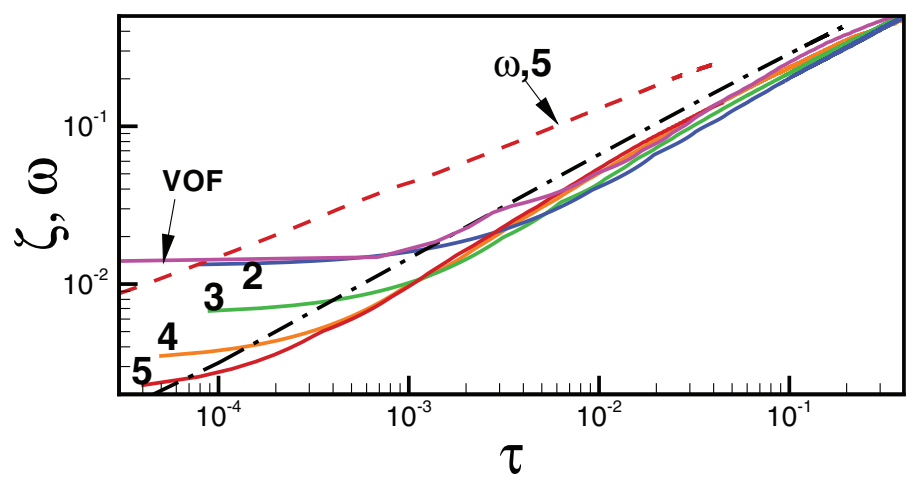

FIG. 4. Simulation results from a DI method and a VOF method for $O h=0.0025, \theta=70^{\circ}$. Coloured solid curves labelled with numbers represent time evolution of the bridge height and are from a DI method with different levels of adaptive mesh refinement (mesh resolutions close to the interface are $2: 1 / 40,3: 1 / 80,4: 1 / 160,5: 1 / 320)$. Purple curve marked VOF: VOF method with mesh resolution of 1/50; dashed-dotted black curve: theoretical result from Eq. (2), found to have a slope very close to $2 / 3$. Red dashed line represents temporal evolution of the bridge width $(\omega=W / R \sin \theta)$ obtained from a DI method using a level-5 mesh, found to have a slope close to $1 / 2$.

for four simulations using the DI method with different numbers of level of refinement. The bridge height in early coalescence is seen to relatively insensitive to mesh resolution after a very short transient that is caused by the finite numerical resolution used, but the duration and significance of which reduce upon grid refinement. We also find that the result obtained from the VOF method agrees very well with the DI results. We present the theoretical result from the simple force-balance model (Eq. (2)) in Fig. 4, which shows a slope of 2/3. A 1/2 regime is almost not visible for $\theta=70^{\circ}$ for the reason discussed above. It is seen that the results obtained with the two $3 \mathrm{D}$ methods are all close to the theoretical prediction (Eq. (2)), and both exhibit a $\zeta \sim \tau^{2 / 3}$ range of at least one decade. It is also noteworthy that Eq. (2) does not contain any adjustable parameter except for the initial value of $\zeta$, which, if sufficiently small, does not affect the result shown in the figure. But this is under the assumption that the dominant curvature radius is exactly equal to $L$. A horizontal shift of the solution of Eq. (2) in Fig. 4 to capture the 3D simulation results would require a proportionality constant for the driving curvature radius that is well within the range 1-5 for various contact angles.

We have also investigated the corresponding growth rate of the width of the bridge during rapid coalescence. A prior experimental study ${ }^{7}$ has confirmed that at low values of $\mathrm{Oh}$, results for the growth of the width are consistent with those from Ref. 4 for coalescence of near-perfectly wetting droplets under creeping-flow conditions: $W \sim t^{1 / 2}$, on the viscous/capillary timescale $\mu R / \gamma$. In Ref. 4, this result is demonstrated to follow from a mass balance over the liquid bridge. This argument does not require input regarding the bridge height vs time. But it does assume that the velocity at which liquid is drawn into the bridge remains constant, and uses Tanner's law ${ }^{17}$ to determine the time constant. In Fig. 4, we also present the time evolution of the bridge width and find that it grows as $\tau^{1 / 2}$, which is consistent with Ref. 7 . We have verified that the average flow speed into the liquid bridge remains unchanged during a considerable time span, which suggests that the mass balance model for the bridge width also applies very well even for inertial coalescence. Although there would here be an opportunity to extend the analysis of Ref. 4 to non-flat droplets by using an extension of Tanner's ${ }^{17}$ law, the curvature radius of the moving contact line is very small, the effect of which on the contact-line dynamics still remains unclear.

We have conducted simulations for droplet coalescence with different contact angles and find that the width of the connecting bridge grows according to $\omega \sim \tau^{1 / 2}$ for all cases during the inertial regime over which we have studied the growth of the bridge height in the above. For a wide range of contact angles (but well below $90^{\circ}$ ), the bridge height always grows as $\zeta \sim \tau^{2 / 3}$ in an early stage of coalescence, which is evidenced by the results obtained from the two independent methods, shown in Fig. 5. The theoretical results from Eq. (2) are not included to keep the figure easily readable; the agreement between the simulation results and Eq. (2) is similar to that in Fig. 4. 


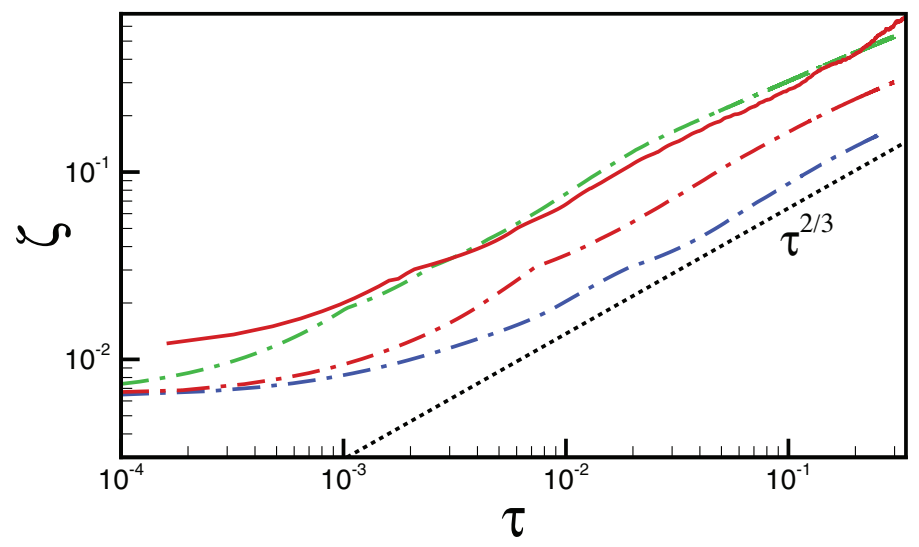

FIG. 5. Temporal evolution of bridge height for different contact angles. The dashed-dotted curves are from a DI method for $\theta=30^{\circ}, 45^{\circ}$, and $80^{\circ}$ (from bottom to top: blue, red, and green, respectively) with $O h=0.0018$. The (red) solid line is from a VOF method with $\theta=45^{\circ}$ and $O h=0.0025$.

From the theoretical model Eq. (2), a transition from a $2 / 3$ to a $1 / 2$ slope is only visible when the contact angle is close to $90^{\circ}$ (Fig. 2). To confirm this with the full numerical simulations is challenging, because the large initial curvature must be resolved. But in Fig. 6, the results are seen to be consistent with the change in slope predicted by the model, albeit that quantitative agreement between the numerical simulation and the theory is achieved only for a rather narrow time range. Thus, we can conclude on this basis together with Fig. 5 that for most contact angle values, in the inertial regime, the height of the liquid bridge grows at an early stage according to a power law in time with a universal exponent of $2 / 3$.

We have also carried out simulations for a larger Ohnesorge number value, $O h=0.1$, where viscous effects become important. The numerical simulation results obtained from both methods show significant deviation from the theoretical prediction for inertial coalescence as in Fig. 7: the power-law exponent becomes considerably larger than $2 / 3$ and is approximately 0.88 , falling in the range measured by Lee et al., ${ }^{6}$ and is also close to the predictions of Narhe et al. ${ }^{5}$ and HernándezSánchez et al. ${ }^{8}$

Different power-law time dependencies have been identified for the height and width of a liquid bridge during inertial coalescence of two identical droplets on a horizontal partially wetting substrate. The liquid bridge height generally exhibits two power-law regimes with different exponents, wherein one regime vanishes for a contact angle of $90^{\circ}$. A simple model has been proposed that is supported by fully 3D numerical simulations using two independent numerical methods.

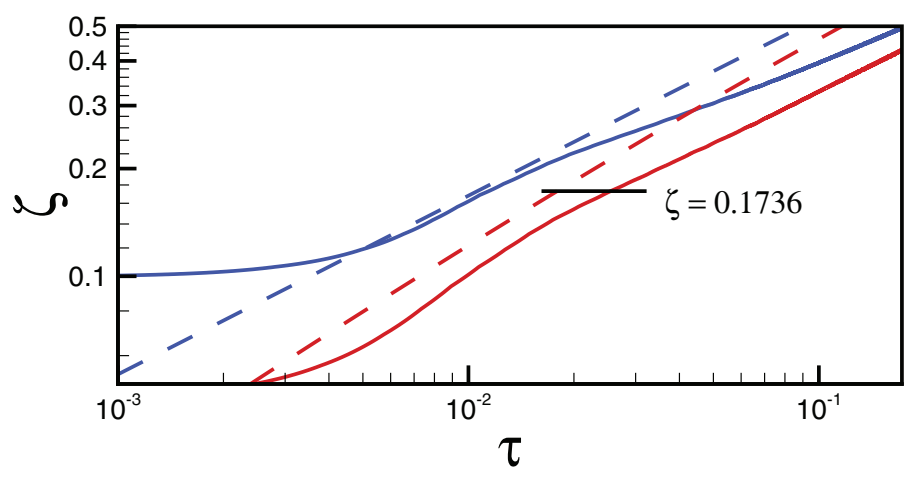

FIG. 6. Transition from a 2/3 regime to $1 / 2$ regime. The solid lines represent simulations using the DI method, the dashed lines Eq. (2); blue (upper two) curves are for $\theta=90^{\circ}$, red (lower two) lines are for $\theta=85^{\circ}$. Horizontal line labels $\zeta=\sin 2 \theta$ for $\theta=85^{\circ}$. Oh $=0.0018$. 


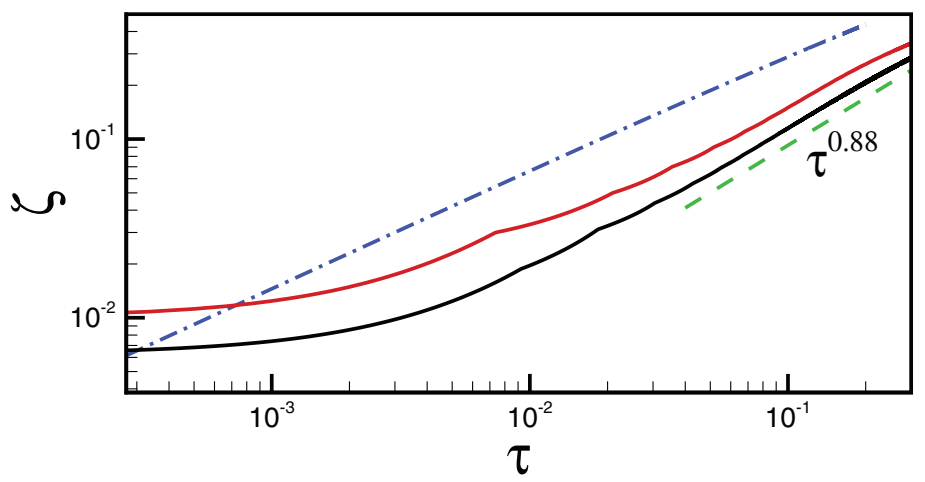

FIG. 7. Simulation results for $O h=0.1, \theta=70^{\circ}$. Lower (black) solid line is from a DI method with 3 levels of adaptive mesh refinement; the upper (red) solid line is from a VOF method with mesh resolution of 1/50. The dashed-dotted (blue) line represents a numerical solution of Eq. (2). The green dashed line represents $\tau^{0.88}$.

The disparity in growth rates of the width and height of the liquid bridge between coalescing droplets on a partially wetting substrate has implications on subsequent flow behaviour. The end state observed in experiments of a "peanut" shape ${ }^{7}$ may readily be attributed to contact-angle hysteresis: the parts of the contact line furthest removed from the liquid bridge does not recede to allow the formation of the otherwise expected single spherical cap end state. But the present results suggest a further mechanism that arises from competition between the two radii of curvature of the liquid bridge. The radius of curvature in the $(x, z)$ plane $R_{x z}$ drives the coalescence as long as it is smaller than that in the $(y, z)$ plane $R_{y z}$.

During the inertial coalescence regime, $R_{x z}$ evolves on the inertial/capillary timescale. It can be estimated by first noticing that the first term in brackets in Eq. (2) is proportional to $L$. In Eq. (3), this has been approximated by two leading terms in a Taylor series expansion, which has been used in the above to establish power-law regimes for the bridge height as a function of time: retaining only the linear term $\zeta / \tan \theta$ results in $H \sim R \tau^{2 / 3}$, which then leads for consistency to $R_{x z}$ $\approx L(H) \sim H / \tan \theta$. Conversely, if the $O\left(\zeta^{2}\right)$ dominates in the first term in brackets in Eq. (3), we have seen that $H \sim R \tau^{1 / 2}$, which results in $R_{x z} \approx L(H) \sim\left(H^{2} / R\right)\left(1+1 / \tan ^{2} \theta\right) . R_{y z}$ can be estimated from instantaneous values of $H \approx R \tau^{\alpha}$ and $Y \approx R(\tau / O h)^{1 / 2}$ by fitting a parabolic interface shape at $x=0, z \approx\left(1-(y / Y)^{2}\right) H$, which gives $R_{y z} \approx Y^{2} / H \approx R \tau^{1-\alpha} / O h$, with $\alpha=1 / 2$ (at late times or if $\theta=90^{\circ}$, as per our results presented above) or $2 / 3$. We expect the coalescence process to be interrupted once $R_{x z}$ is about to overtake $R_{y z}$. Based on the above curvature estimates, this would occur when $\tau>O\left(1 / O h^{3}\right)$ if the bridge height grows following $\tau^{2 / 3}$, and when $\tau>O\left(1 / O h^{2}\right)$ if the height is growing in the $1 / 2$ regime. This indicates that at relatively large values of $O h$, the coalescence process is terminated due to the resisting curvature in the $(y, z)$ plane becoming dominant. We have indeed found in our 3D numerical simulations that a peanut shape can be obtained at a large value of $\mathrm{Oh}$ for a fixed window of contact-angle hysteresis: for a contact-angle hysteresis window of [50 $71^{\circ}$ ], a spherical cap was obtained at $O h=0.0025$, whereas a peanut-type shape was observed for $O h=0.01$ (in both cases, a DI method was used). Evidently, further detailed computational or experimental studies would be required to establish the precise relation between the initial stages of coalescence investigated here and the final droplet shape, as well as the relative importance of contact-angle hysteresis and the mechanism proposed here.

We have extended Eq. (2) to the asymmetrical coalescence of two droplets with different sizes $R_{1}$ and $R_{2}$ on a substrate. The radius of interface curvature $L$ can be evaluated by

$$
\begin{aligned}
L(z)= & \frac{1}{2}\left[R_{1} \sin \theta-\left(R_{1}^{2}-\left(z+R_{1} \cos \theta\right)^{2}\right)^{1 / 2}\right. \\
& \left.+R_{2} \sin \theta-\left(R_{2}^{2}-\left(z+R_{2} \cos \theta\right)^{2}\right)^{1 / 2}\right] .
\end{aligned}
$$


Using $R_{1} \sin \theta$ as the length scale, we arrive at a normalized pressure-balance equation that generalizes Eq. (2). After a Taylor series expansion, keeping the first and second order terms of $\zeta$, we have

$$
\left(\frac{\zeta}{\tan \theta}+\frac{\zeta^{2}}{2}\left(1+\frac{1}{\tan ^{2} \theta}\right) \frac{R_{1}+R_{2}}{2 R_{2}}\right)\left(\frac{d \zeta}{d \tau}\right)^{2}=1
$$

It is seen that the leading order result does not depend on the size ratio of the two droplets, which means the $2 / 3$ regime reported above is still expected for the coalescence of non-equal sized droplets.

Finally, the main validation of the theory presented herein is through numerical simulations. A comparison against prior experiments has also been made, but these data from prior studies turn out to be for a range of contact angles that is too limited, as these do not include results (over several orders of magnitude of the bridge height and width) for contact angles close to $90^{\circ}$, which would be essential, in view of the results obtained herein.

Y.S. acknowledges the SEMS/QMUL start-up grant for new academics. The simulations were performed using the high-performance computer clusters provided by the Queen Mary University of London and those of CINES/Jade (c2011026679, c2012026893, and c20132b6893). M.M. and D.L. would like to thank the financial support from a Marie Curie Early Stage Research Training Fellowship of the European Community Sixth Framework Programme under the Contract No. MEST-CT-2005-020426.

${ }^{1}$ J. Frenkel, "Viscous flow of crystalline bodies under the action of surface tension," J. Phys. (Moscow) 9, 385 (1945).

${ }^{2}$ V. S. Nikolayev, D. Beysens, and P. Guenoun, "New hydrodynamic mechanism for drop coarsening,” Phys. Rev. Lett. 76, 3144 (1996).

${ }^{3}$ S. K. Cho, H. Moon, and C.-J. Kim, "Creating, transporting, cutting, and merging liquid droplets by electrowetting-based actuation for digital microfluidic circuits," J. Microelectromech. Syst. 12, 70-80 (2003).

${ }^{4}$ W. D. Ristenpart, P. M. McCalla, R. V. Roy, and H. A. Stone, "Coalescence of spreading droplets on a wettable substrate," Phys. Rev. Lett. 97, 064501 (2006).

${ }^{5}$ R. Narhe, D. Beysens, and Y. Pomeau, "Dynamic drying in the early-stage coalescence of droplets sitting on a plate," Europhys. Lett. 81, 46002 (2008).

${ }^{6}$ M. W. Lee, D. K. Kang, S. S. Yoon, and A. L. Yarin, “Coalescence of two drops on partially wettable substrates," Langmuir 28, 3791-3798 (2012).

${ }^{7}$ N. Kapur and P. H. Gaskell, "Morphology and dynamics of droplet coalescence on a surface," Phys. Rev. E 75, 056315 (2007).

${ }^{8}$ J. F. Hernández-Sánchez, L. A. Lubbers, A. Eddi, and J. H. Snoeijer, "Symmetric and asymmetric coalescence of drops on a substrate," Phys. Rev. Lett. 109, 184502 (2012).

${ }^{9}$ J. Eggers, J. R. Lister, and H. A. Stone, "Coalescence of liquid drops,” J. Fluid Mech. 401, 293-310 (1999).

${ }^{10}$ Y. Sui, H. Ding, and P. D. M. Spelt, "Numerical simulations of flows with moving contact lines," Annu. Rev. Fluid Mech. 46, 97-119 (2014).

${ }^{11}$ H. Ding, P. D. M. Spelt, and C. Shu, "Diffuse interface model for incompressible two-phase flows with large density ratios," J. Comput. Phys. 226, 2078-2095 (2007).

${ }^{12}$ H. Ding and P. D. M. Spelt, "Onset of motion of a three-dimensional droplet on a wall in shear flow at moderate Reynolds numbers," J. Fluid Mech. 599, 341-362 (2008).

${ }^{13}$ Y. Sui and P. D. M. Spelt, "Validation and modification of asymptotic analysis of slow and rapid droplet spreading by numerical simulation," J. Fluid Mech. 715, 283-313 (2013).

${ }^{14}$ Y. Sui and P. D. M. Spelt, "An efficient computational model for macroscale simulations of moving contact lines," J. Comput. Phys. 242, 37-52 (2013).

15 J.-B. Dupont and D. Legendre, "Numerical simulation of static and sliding drop with contact angle hysteresis," J. Comput. Phys. 229, 2453-2478 (2010).

${ }^{16}$ D. Legendre and M. Maglio, "Numerical simulation of spreading drops," Colloids Surf., A 432, 29-37 (2013).

${ }^{17}$ L. H. Tanner, "The spreading of silicone oil drops on horizontal surfaces," J. Phys. D 12, 1473-1484 (1979). 